\title{
The Italian contribution to the Gaia data processing and archiving
}

\author{
Michele Martino ${ }^{1}$, Armando Ciampolini ${ }^{1}$, Rosario Messineo ${ }^{1}$, Angelo \\ Mulone $^{1}$, Enrico Pigozzi ${ }^{1}$, Vilma Icardi ${ }^{1}$, Filomena Solitro ${ }^{1}$, \\ Marco M. Castronuovo ${ }^{2}$, Mario G. Lattanzi ${ }^{3}$, Roberto Morbidelli ${ }^{3}$, \\ Ronald Drimmel $^{3}$, Maria Sarasso ${ }^{3}$, Deborah Busonero ${ }^{3}$, \\ Mariateresa Crosta ${ }^{3}$, Daniele Gardiol ${ }^{3}$, Alberto Vecchiato ${ }^{3}$ \\ and the Italian Gaia Team ${ }^{3,4,5,6,7,8}$ \\ 1 ALTEC S.p.A \\ email: michele.martino@altecspace.it \\ 2 ASI Agenzia Spaziale Italiana \\ ${ }^{3}$ INAF - Osservatorio Astronomico di Torino \\ ${ }^{4}$ INAF - Osservatorio Astronomico di Padova \\ ${ }^{5}$ INAF - Osservatorio Astronomico di Bologna \\ ${ }^{6}$ INAF - Osservatorio Astronomico di Roma-Teramo \\ ${ }^{7}$ INAF - Osservatorio Astronomico di Napoli \\ ${ }^{8}$ INAF - Osservatorio Astrofisico di Catania
}

\begin{abstract}
Gaia is an ESA Cornerstone mission, scheduled to be launched in spring 2013, dedicated to precisely measure the positions and motions of over a billion stars in our galaxy: the Milky Way. Gaia Data Processing Center Turin (DPCT), the Italian DPC, is hosted and operated at ALTEC in Turin. The primary objective of DPCT is to provide the infrastructure and operations support to the Astrometric Verification Unit (AVU) activities for CU3 and the Italian participation to the Gaia data processing tasks. DPCT will archive all of the data , produced for and delivered to DPAC as part of the Italian contribution to the activities of CU4, CU5, CU7, and CU8.
\end{abstract}

Keywords. astrometry, surveys, catalogs

\section{Gaia Mission}

The Gaia mission aims to obtain astrometric data with a precision two hundred times greater than that of Hipparcos and astrophysical information on brightness in different spectral bands that will allow studying in detail the formation, dynamics, chemistry and evolution of our galaxy. It will also be possible to detect extrasolar planets and observe asteroids, galaxies and quasars. Gaia satellite is completely developed by ESA, including also the scientific instruments. The contribution of member states is devoted to the scientific program. Italy is the third contributor country.

\section{Gaia DPCT}

Gaia DPCT (see Fig. 1 for one of its constituting elements) is hosted and operated at ALTEC centre in Turin. The main objective of DPCT is to provide the infrastructure (in terms of HW, SW and communications) and operations support to the Astrometric Verification Unit (AVU) activities for CU3 and the Italian participation to the Gaia 
data processing tasks. DPCT will archive all of the data (photometric calibration data, libraries of spectra, etc) produced for and delivered to DPAC as part of the Italian contribution to the activities of CU4, CU5, CU7, and CU8. AVU is the unit responsible for the development and maintenance of the following CU3 software products: AIM - Astrometric Instrument Model, in charge of processing the Astro data telemetry in order to monitor and analyse the Astro instrument response over the mission lifetime. BAM/AVU - Basic Angle Monitoring software system, in charge of processing the BAM device telemetry in order to monitor and analyse the BA behaviour over time. GSR Global Sphere Reconstruction, the mathematical and numerical framework that shall be used to verify the global astrometric results produced by AGIS. In addition the DPCT will provide hosting and operations of the GAia Relativistic Experiment on Quadrupole experiment (GAREQ) and of Initial Gaia Source List (IGSL) database. The IGSL DB will be maintained and upgraded throughout the Gaia operational lifetime and will be used to produce upgraded version of the IGSL catalogue to be delivered periodically to ESAC and ingested in the Main DB.

\section{Ground Segment}

The Gaia mission requires, as a critical element, the construction of a Ground Segment (GS) that shall be operative for all the duration of the mission and, subsequently, up to the achievement of the expected quality before the result publication and distribution to the international scientific community. The GS is composed of six different Data Processing Centres (DPCs) and its implementation and management is the primary task of the European consortium DPAC, responsible for ESA of the reduction of data of the mission.

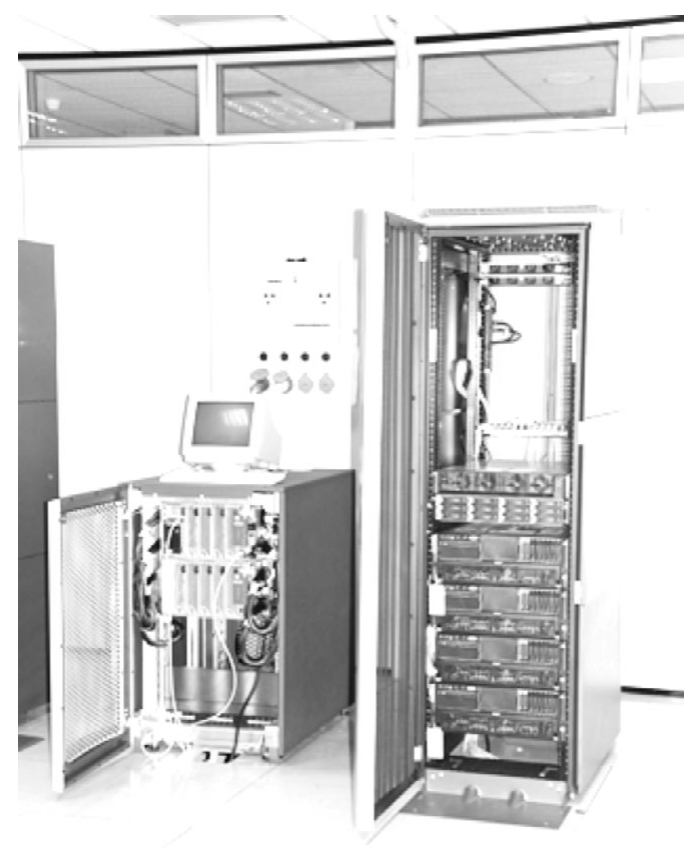

Figure 1. DPCT Development \& Test Platform 
The six centers are: ESAC/DPCE, CNES/DPCC, Barcelona/DPCB, Cambridge/ DPCI, Geneva/DPCG and Turin/DPCT. The responsibility for reducing the enormous amount of data that will be produced by the mission is entrusted to the DPAC (Data Processing and Analysis Consortium), a consortium of European research institutions created in response to an ESA Announcement of Opportunity. The DPAC is structured into 8 Coordination Units $(\mathrm{CU})$, each responsible for a particular aspect of data processing. INAF-OATO is responsible for the definition, design, development, test and validation of the software for all AVU subsystems, GAREQ and the IGSL that shall be delivered to DPCT. It is also responsible for all of the coordination activities necessary for the development and operations of the DPCT. ALTEC is responsible for the definition, design, implementation, test, validation, and operations of the infrastructure of the DPCT that will be hosted on their premises and of the integration, testing and operations (science operations excluded) of the AVU, GAREQ and IGSL delivered software. 